\title{
Minimally invasive cardiac surgery—a push to kaizen!
}

\author{
Om Prakash Yadava ${ }^{1}$
}

Received: 3 January 2018 / Accepted: 8 January 2018 / Published online: 8 February 2018

(C) Indian Association of Cardiovascular-Thoracic Surgeons 2018

The advent of cardiopulmonary bypass (CPB) in 1953 brought most intracardiac structural disorders of the heart within the purview of cardiac surgery. The success of 1960s elevated the cardiac surgeons to the 'Godly Pedestal', and he became the undisputable numero uno of the medical fraternity. The 'holier than thou' image, the 'cash-cow' status of the speciality, sprinkled with the haughtiness and arrogance of being a cardiac surgeon, and topped with the exclusivity of treatment domain for most cardiac illnesses, there being no interventional cardiology then, led to a kind of an inertia and the surgeons sat on their haunches in 1970s and 1980s with no great innovations or developments in the field. The entire fraternity, however, was shaken up with a rude jolt with the advent of percutaneous interventions for coronary artery disease. Soon followed the device closure for left to right shunts and the turf seemed to be slipping under the sleeping giants' lair. That got the cardiac surgical fraternity thinking and thus sprung the sub-speciality of minimally invasive cardiac surgery (MICS), not honestly for the felt needs of the patients, but of the treating doctors ....hic...cardiac surgeons.

The first forays into MICS-mitral valve surgery were simultaneously reported by Navia and Cosgrove [1] and Cohn et al. [2] and into aortic valve surgery by Cosgrove and Sabik [3] in mid 1990s. There after the speciality blossomed, and sequentially video assistance was introduced by Carpentier et al. [4], transthoracic aortic clamping by Chitwood et al. [5], port-access by the Leipzig group headed by Mohr et al. [6] and robotic computer assisted surgery by once again Carpentier et al. [7]. As we stand today, most intracardiac pathologies have a parallel minimally invasive or transcatheter solution to the standard sternotomy, open-chest route. Newer hard ware for peripheral cannulation, development of suture-less prosthesis and transcatheter options, improvement in robotic arms and video

Om Prakash Yadava

op_yadava@yahoo.com

1 National Heart Institute, 49-50, Community Centre, East of Kailash, New Delhi 110065, India assistance, strobe lighting systems, newer instrumentation and, the most important of all, the thought process - the willingness and readiness to perform these procedures, saw MICS gaining traction. Infact, in some centres across the world, MICS is a default strategy for all cardiac surgeries and sternotomy option is taken anecdotally by design. MICS thus seems to be finding favours with the patients, the doctors as also the industry, which is willing to sponsor it. This amalgam indeed is rare.

No questions and no denial - the proof of the concept, the feasibility and, to some extent, improved soft end points have been demonstrated. It's a no brainer that MICS has a cosmetic advantage, reduces blood loss and length of the stay in the hospital and avoids the potentially devastating infective problems of mediastinitis/sternal dehiscence besides reducing the post-operative recovery time. But is that enough? The questions that beg an answer are as follows: Do MICS improve cardiac outcomes? Can it be taught with a reasonable learning curve without compromising patient's interests? Are these procedures for the real world or should they be limited to a select few centres and in the hands of the blessed and the gifted mavericks, for minimal access should not translate into maximising technical difficulties. In India, it also raises a surrogate question of the everlasting debate of relevance of science to the society-Do the human and fiscal resources required for these surgeries justify their propagation in developing and impoverished countries?

Just as we push for these technologies, we must demonstrate hard outcomes in terms of survival benefit and not just pain relief and early return to work-indeed very soft end points. I am acutely seized of the fact that the naysayers may argue that the kind of spectacular advances that cardiac surgery has seen over a short period of 50 years would have been nigh impossible, if every single new procedure was to be introduced only after long-term outcome analysis - a point well taken. But then, a counter argument can be that when we introduced closure of ASD and VSDs in 1950s, there was no other option and therefore there was no need to demonstrate the outcome analysis at that time. Similarly, the valves in the 1960s and the coronaries in 1970s had no option, 
but today, we have very good, well demonstrated and established evidence-based procedures, in terms of conventional sternotomy and CPB-based open heart surgeries as well as percutaneous options for coronaries, valves and left to right shunts. Therefore, if today, we have to introduce a disruptive new technology, then we are morally bound to demonstrate, in clear cut terms, superiority and not just inferiority or equivalence. Thus, these arguments fall flat in contemporary relevance. I don't want to sound as an impediment to science. To the contrary, I want to push the envelope and the proof of the pudding is our dedicating a full 'Featured Issue' on this subject, yet we should also be aware and cognizant of the admonition of Mark Twain, 'We should keep an open mind, but not so open that the brains fall out'. So, even if we don't subject these new procedures to randomised controlled trials, yet they must be subjected to diligent scientific scrutiny, done with rigour and neutrality, and sans any conflicts of interest. We therefore need bench marks not only for judging the surgeon's performance, but also because they are going to be increasingly demanded by the regulatory authorities, insurance companies and the payers. Sooner, we establish them and live up to them the better it will be for us. What we need thus is a neutral ombudsman who dispassionately examines all data and sets definitive hard end points, the foremost being event free survival. Only if we score favourably here, should the entire surgical fraternity take to MICS with vengeance.

I grant some of these questions are now being answered with the development of transcatheter solutions to valvular heart disease. Infact, D'onofrio and Gerosa have coined a new terminology 'Micro-invasive Cardiac Surgery' to cover these groups [8]. Even though these therapies today have been proven as class I indication for only inoperable and surgical high risk patients, but it would be naive and foolhardy on our part to believe that this push towards kaizen is not going to move towards lower risk cases. We therefore need to get out of our denial mode and not only accept the reality but adopt the new technology and sooner the better.

We also need to rethink our strategy of training and curricula need to be redrawn to include wire skills and hand-eye coordination skills for video-assisted surgeries. We need to have a new breed of 'cardiologico-surgical' doctors who should be the single window solution to the patients' problems as the distinction line between the cardiologist and the surgeon seems to be blurring. 'We need to understand that this is not a futuristic visionary scenario - it is already happening, and it is a reality' [8]. It is this amalgamation that was highlighted by the German Cardiology Society electing a surgeon-Prof. Friedrich Mohr as its President and the German Cardiac Surgical Society electing, Prof. Christian W. Hamm, a cardiologist, as its President, thus underlining the motto 'ein Herzein Team' meaning 'One heart-one Team'.

This collaboration to the point of even amalgamation is all the more relevant to us in India. As its stands today in most centres in this country, what treatment a patient gets depends on who sees the patient first. A Gruntzig type A, mid LAD lesion, can end up on the operating table and a type $\mathrm{C}$ heavily calcified irregular and ragged distal bifurcation left main stenosis with a circumflex arising at a $90^{\circ}$ angle may end on the catheterisation table, if an aggressive interventional cardiologist happens to be the first point of contact. No regulation and no level of formulation of guidelines will be able to control this basic human tendency of self-preservation, more so in a scenario of reducing patient volume per centre, as the centres burgeon even in tier 3 cities. We will be able to deliver ethical and patient-centric decisions only if we develop an attitude of collaboration and a patient is handled by a doctor who has skills and proficiency in all available treatment forms, so that the personal pecuniary and other considerations are nullified.

The training programmes should be simulation-based and only after a pre-defined benchmark proficiency is achieved should honing of skills be allowed on live patients. However, our training infrastructure is limited and there are hardly a few skills laboratories with advanced simulationbased teaching facilities in the country. Therefore, we will have to be choosy and instead of the heads of the departments and the seniors, the sixty plus of my kind jumping the queue to be on the top to register for these training programmes, we should encourage the youngsters, the kind of 40 plus group, who have sufficient experience of conventional surgery and yet have a sizeable surgical career ahead of them, who also have the zest and the verve and the youthful vibrancy to learn new techniques. Only once we have trained our youth and we have vacancies in these training programmes, with no young takers, those seats should be allotted to the 60 years plus.

Are these niche areas? My categorical answer-Yes. It's therefore of paramount importance that the MICS is being advised, by the 'right doctor' and that too for the 'right patient'. It's also important that those who take to MICS should have commitment to the speciality and be convinced that it has got positive outcomes to the patients and not just take to it for the reason of joining the bandwagon and for survival in the competitive modern world. They should not take it as a compulsion, driven and sucked into it, but should take to the speciality positively and pro-actively.

A caveat for our senior colleagues - don't compromise patient interest for reasons of ego and if you are not adequately trained, conventional surgery still rules the roost and we should not offer it to the patients apologetically, but with a firm conviction that under the circumstances, what we are offering to our patients is the best in their interest. Infact, even conventional surgeons can take a lesson or two from our MICS counterparts, in terms of value of reducing tissue trauma during sternotomy, handling the tissues gently, use of lower settings of energy for electro-cautery and not over diathermising the tissues, avoiding over retraction and fracture of sternum, ribs and dislocation of costo-chondral junctions, 
early removal of central lines, urinary catheters and chest tubes, judicious use of fast track protocols and meticulous planning and execution of the surgery, so that it is expediently performed. This diligently pursued may make the results of conventional surgery better and even quite akin to the MICS outcomes.

When the time comes of a good idea, it cannot be bridled or subjugated by any means and sure enough minimally invasive and micro invasive cardiac surgery shall be the buzz words moving forward. We are thus at a critical and strategic inflection point in the evolution of cardiac surgery. It has been challenged both by our cardiology counter parts, as also a self-challenge with in the cardiac surgical community, more as a survival response than anything else. We thus need to evolve continuously and keep reinventing ourselves rather than repeat the mistake of 1970s and 1980s of developing the 'inertia of success'. But by the same token, our response should not be that of desperation and dejection of possible extinction. As one avenue closes, new vistas shall open up, as Doris Day will have you believe,

\section{Que Sera, Sera}

Whatever will be, will be,

the future's not ours to see,

Que sera, sera.

So, instead of bemoaning the slipping turf, encourage the youngsters in the team to move forwards. Thankfully, I have passed my time, gleefully and with ample measure of satisfaction with the good old 'Heart-Lung' machine through the sternotomy option, but can't help but acknowledge and applaud the new developments.

It's in the acceptance of this reality that we are coming out with a featured issue of the Indian Journal of Thoracic and Cardiovascular Surgery on minimally invasive cardiac surgery and it's my singular privilege to present Dr. Johannes Bonatti, Chief of Cardiac Surgery, Cleveland Clinic, Abu Dhabi, as our honoured Guest Editor.

\section{References}

1. Navia JL, Cosgrove DM. Minimally invasive mitral valve operations. Ann Thorac Surg. 1996;62:1542-1544.

2. Cohn LH, Adams DH, Couper GS, et al. Minimally invasive cardiac valve surgery improves patient satisfaction while reducing costs of cardiac valve replacement and repair. Ann Surg. 1997;226:421-428.

3. Cosgrove DM 3rd, Sabik JF. Minimally invasive approach for aortic valve operations. Ann Thorac Surg. 1996;62:596-597.

4. Carpentier A, Loulmet D, Carpentier A, et al. Open heart operation under videosurgery and minithoracotomy - First case (mitral valvuloplasty) operated with success. CR Acad Sci. 1996;319:219-223.

5. Chitwood WR Jr, Elbeery JR, Chapman WH, et al. Video-assisted minimally invasive mitral valve surgery: the 'micro-mitral' operation. J Thorac Cardiovasc Surg. 1997;113:413-414.

6. Mohr FW, Falk V, Diegeler A, Walther T, van Son JA, Autschbach R. Minimally invasive port-access mitral valve surgery. J Thorac Cardiovasc Surg. 1998;115:567-576.

7. Carpentier A, Loulmet D, Aupècle et al. Computer assisted open heart surgery-First case operated on with success. CR Acad Sci III. 1998;321:437-442.

8. D'Onofrio A, Gerosa G. Shifting at paradigm cardiac surgery: from minimally invasive to micro invasive. J Heart Valve Dis. 2015;24: 528-30. 